\title{
Verzeichnis der Abkürzungen
}

\begin{tabular}{|c|c|c|c|}
\hline $\begin{array}{l}\mathbf{A A} \\
\mathbf{A A A}\end{array}$ & $\begin{array}{l}\text { Acta Astronomica } \\
\text { Asociación Argentina de } \\
\text { Astronomla }\end{array}$ & BAB & $\begin{array}{l}\text { Bulletin Astronomique de } \\
\text { l'Observatoire Royal de Bel- } \\
\text { glque }\end{array}$ \\
\hline AAS & American Astronomical Society & BAC & Bulletin of the Astronomical \\
\hline AAVSO & $\begin{array}{l}\text { American Association of } \\
\text { Variable Star Observers }\end{array}$ & BAN & $\begin{array}{l}\text { Institutes of Czechoslovakia } \\
\text { Bulletin of the Astronomical }\end{array}$ \\
\hline Abh. & Abhandlungen & & Institutes of the Netherlands \\
\hline Abstr. & Abstract & BAV Rundbrief & Mittellungsblatt der Berliner \\
\hline Abt. & Abteilung & & Arbeitsgemeinsehaft für Ver- \\
\hline $\mathbf{A C}$ & $\begin{array}{l}\text { Astronomisches Zirkular der } \\
\text { UdSSR }\end{array}$ & Beob. & $\begin{array}{l}\text { anderliche Sterne } \\
\text { Beobachtungen etc. }\end{array}$ \\
\hline Acad. & Academy etc. & Ber. & Berichte \\
\hline $\begin{array}{l}\text { Accad. } \\
\text { AFCRI. }\end{array}$ & $\begin{array}{l}\text { Accadémia } \\
\text { Air Force Cambridge Research } \\
\text { Laboratories }\end{array}$ & BIA & $\begin{array}{l}\text { Bulletin des Instituts für } \\
\text { Theoretische Astronomle, } \\
\text { Leningrad }\end{array}$ \\
\hline $\mathbf{A G}$ & Astronomische Gesellschaft & BMI & Bulletin de Mesures \\
\hline AIAA J & $\begin{array}{l}\text { American Institute of Aero- } \\
\text { nautics and Astronautics. } \\
\text { Journal }\end{array}$ & $\begin{array}{l}\text { Bol. } \\
\text { BSAF }\end{array}$ & $\begin{array}{l}\text { Ionosphériques } \\
\text { Boletín } \\
\text { L'Astronomie. Bulletin de la }\end{array}$ \\
\hline $\mathbf{A J}$ & Astronomical Journal & & Sociétó Astronomique de \\
\hline $\mathbf{A J B}$ & Astronomischer Jahresbericht & & France \\
\hline AJ UdSSR & $\begin{array}{l}\text { Astronomisches Journal der } \\
\text { UdSSR }\end{array}$ & $\begin{array}{l}\text { Bull. } \\
\text { Bull hor }\end{array}$ & $\begin{array}{l}\text { Bulletin } \\
\text { Bulletin horaire }\end{array}$ \\
\hline Akad. & Akademie & Bull KE & Bulletin der Stationen \\
\hline AN & Astronomische Nachrichten & & zur optischen Beobachtung \\
\hline $\begin{array}{l}\text { An. } \\
\text { angew. }\end{array}$ & $\begin{array}{l}\text { Anales etc. } \\
\text { angewandt }\end{array}$ & bzw. & $\begin{array}{l}\text { künstlicher Erdsatelliten } \\
\text { beziehungsweise }\end{array}$ \\
\hline Ann. & Annalen etc. & Ci. & Ciencia \\
\hline ApJ & Astrophysical Journal & Circ. & Circular ete. \\
\hline Arch. & Archiv & Cl. & \\
\hline Ark Astr & Arkiv för Astronomi & Coll. & Collection etc. \\
\hline As. & Asociación & Commun. & Communications \\
\hline ASA & $\begin{array}{l}\text { Astronomical Society of Au- } \\
\text { stralia }\end{array}$ & $\begin{array}{l}\text { Comun. } \\
\text { Contr. }\end{array}$ & $\begin{array}{l}\text { Comunicazioni } \\
\text { Contributions etc. }\end{array}$ \\
\hline ASP & $\begin{array}{l}\text { Astronomical Society of the } \\
\text { Paciflc }\end{array}$ & COSPAR IB & $\begin{array}{l}\text { COSPAR Information Bulle- } \\
\text { tin }\end{array}$ \\
\hline Ass. & Association & CR & Comptes Rendus, Académie \\
\hline Astronaut. & Astronautik etc. & & des Sciences, Paris \\
\hline $\begin{array}{l}\text { Astr. } \\
\text { Astrophys. } \\
\text { Atmosph. }\end{array}$ & $\begin{array}{l}\text { Astronomie etc. } \\
\text { Astrophysik etc. } \\
\text { Atmosphire etc. }\end{array}$ & C.S.I.R.o. & $\begin{array}{l}\text { Commonwealth Scientifle and } \\
\text { Industrial Research Organl- } \\
\text { zation }\end{array}$ \\
\hline B. & Besprechung(en) & DAN & Berichte (Doklady) der Aka- \\
\hline BA & $\begin{array}{l}\text { Bulletin Astronomique } \\
\text { British Astronomical Asso- }\end{array}$ & & demie der Wissenschatten deI \\
\hline BAA & $\begin{array}{l}\text { British Astronomical Asso- } \\
\text { ciation }\end{array}$ & Dep. & $\begin{array}{l}\text { Department } \\
\text { Depseningraa }\end{array}$ \\
\hline BAA Cire & $\begin{array}{l}\text { British Astrononical Asso- } \\
\text { ciation Circular }\end{array}$ & $\begin{array}{l}\text { dgl. } \\
\text { d. h. }\end{array}$ & $\begin{array}{l}\text { desgleichen } \\
\text { das hei } B \text { t }\end{array}$ \\
\hline BAA H & $\begin{array}{l}\text { Britiah Astronomical Asso- } \\
\text { ciation Handbook }\end{array}$ & $\begin{array}{l}\text { Diss. } \\
\text { Div. }\end{array}$ & $\begin{array}{l}\text { Dissertation } \\
\text { Division }\end{array}$ \\
\hline
\end{tabular}




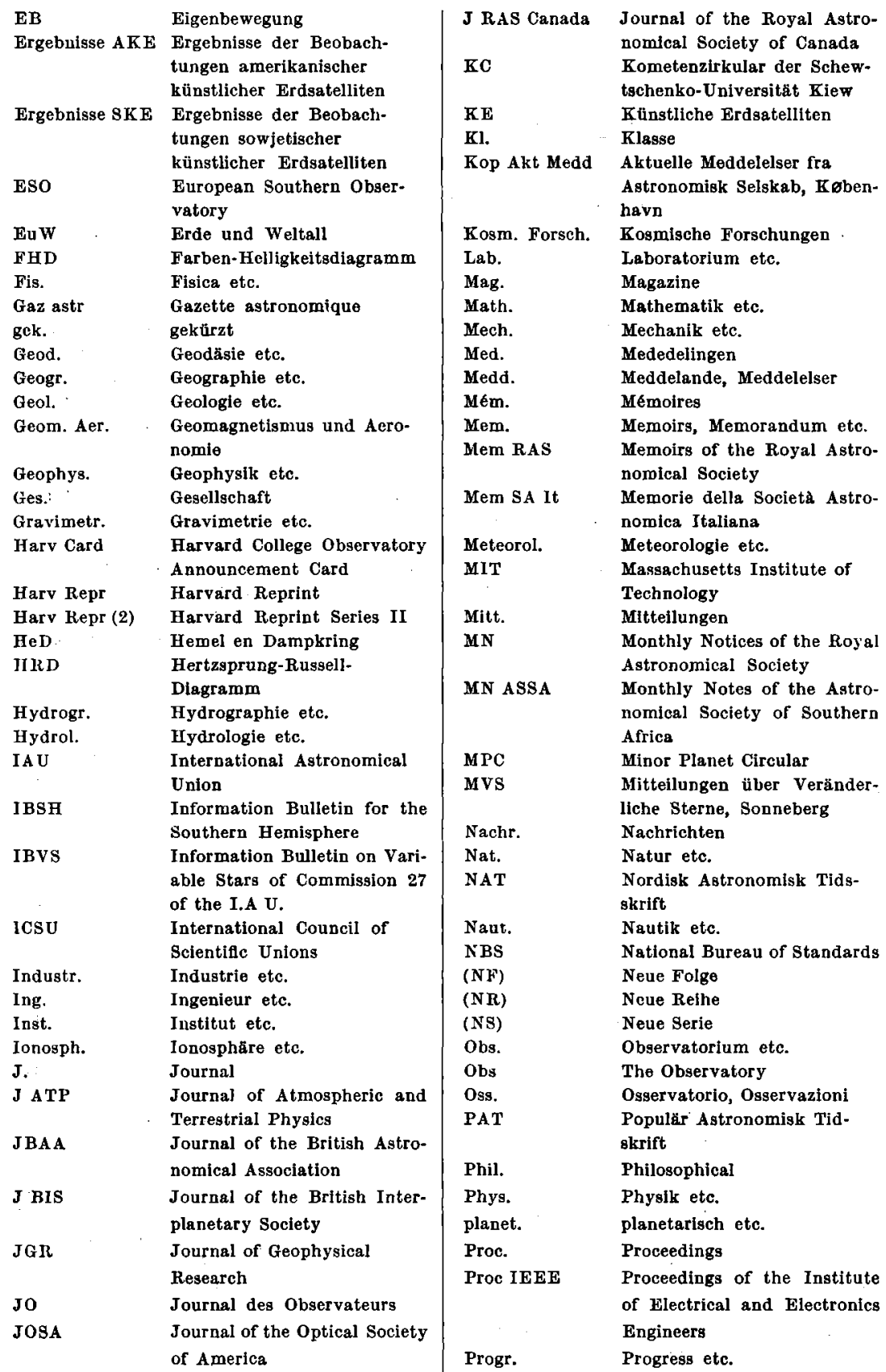


PTA

Pubbl.

Publ.

Publ ASP

Rap.

RAS

Rech.

Ref.

Rend.

Rep.

Repr.

Res.

Rev.

RG

RH

Ric.

RJ UdSSR

R. 0 .

Roy.

SAO

Sci.

Sect.

Ser.

S. I. R.

Sitzber.

Sky Tel.

Soc.

SSR

Sternw.

Stud. Cere.
Polskie Towarzystwo Astro-

nomiczne

Pubblicazioni

Publikationen etc.

Publications of the Astronomical Society of the Paciflc

Raportoj

Royal Astronomical Society

Recherches

Referat

Rendiconti

Report

Reprint

Research

Review etc.

Radialgesch windigkeit

Ríse Hvèzd

Ricerche etc.

Referatjournal. Astronomie,

Geodāsie. Akademie der Wissenschaften der UdSSR

c.S.I.1R.O.

Royal etc.

Smithsonian Institution, Astrophysical Observatory

Science etc.

Section

Serie etc.

Service International Rapide

des Latitudes

Sitzungsberichte

Sky and Telescope

Society etc.

Sozialistische Sowjetrepublik

Sternwarte

Studil și Cercetări
Suppl.

SuW

$\mathbf{T}$

TAB

Techn.

TH

theoret.

Trans.

U., ü.

UAI Circ

UC, Union Circ

Univ.

VdS Nachr.

Verf.

Verh.

Veröff.

vgl.

VJS

vs Bull

WAGO

W. B.

Wet.

Wiss.

Z.

z. B.

ZD

ZfA

ZfMG

( )
Supplement

Sterne und Weltraum

Tidskrift etc.

Tokyo Astronomical Bulletin

Technik etc.

Technische Hochschule

theoretisch etc.

Transactions

Ubersetzung, übersetzt

Union Astronomique Inter-

nationale. Circulaire

Circular of the Union Observatory

Universität etc.

Nachrichtenblatt der Vereinigung der Sternfreunde, Berlin

Verfasser(in)

Verhandlungen

Veröffentlichungen

vergleiche

Vierteljahresschrift

Veränderliche Sterne, Bulletin

Astronomisch-Geodätische

Gesellschaft der UdSSR

Weitere Besprechung(en)

Wetenschappen

Wissenschaften

Zeitschrift

zum Beispiel

Zvaigżnotā Debess

Zeitschrift für Astrophysik

Zentralblatt für Mathematik

und ihre Grenzgebiete

(Serie, Folge, Reihe) 